\title{
Fast atmospheric response to a sudden thinning of Arctic sea ice
}

\author{
Tido Semmler $^{1} \cdot$ Thomas Jung $^{1,2} \cdot$ Soumia Serrar ${ }^{1}$
}

Received: 29 April 2014 / Accepted: 11 April 2015 / Published online: 8 May 2015

(C) The Author(s) 2015. This article is published with open access at Springerlink.com

\begin{abstract}
In order to understand the influence of a thinner Arctic sea ice on the wintertime atmosphere, idealized ensemble experiments with increased sea ice surface temperature have been carried out with the Integrated Forecast System of the European Centre for Medium-Range Weather Forecasts. The focus is on the fast atmospheric response to a sudden "thinning" of Arctic sea ice to disentangle the role of various different processes. We found that boundary layer turbulence is the most important process that distributes anomalous heat vertically. Anomalous longwave radiation plays an important role within the first few days before temperatures in the lower troposphere had time to adjust. The dynamic response tends to balance that due to boundary layer turbulence while cloud processes and convection play only a minor role. Overall the response of the atmospheric large-scale circulation is relatively small with up to $2 \mathrm{hPa}$ in the mean sea level pressure during the first 15 days; the quasi-equilibrium response reached in the second and third month of the integration is about twice as large. During the first few days the response tends to be baroclinic in the whole Arctic. Already after a few days an anti-cyclonic equivalent-barotropic response develops over north-western Siberia and north-eastern Europe. The structure resembles very much that of the atmospheric equilibrium response indicating that fast tropospheric processes such as fewer quasi-barotropic cyclones entering this continental area are key opposed to slower processes such as
\end{abstract}

Tido Semmler

tido.semmler@awi.de

1 Helmholtz Centre for Polar and Marine Research, Alfred Wegener Institute, Am Handelshafen 12, 27570 Bremerhaven, Germany

2 University of Bremen, Bremen, Germany those involving, for example, stratosphere-troposphere interaction.

Keywords Arctic sea ice - Arctic boundary layer . Atmospheric circulation $\cdot$ Numerical modelling

\section{Introduction}

Arctic sea ice has declined substantially over the past decades and record minima in its extent have been reached in September 2007 and 2012 (e.g. Parkinson and Comiso 2013). This raises the question as to whether these changes have far-field impacts on the weather and climate of the Northern Hemisphere middle latitudes (see review papers Budikova 2009; Bader et al. 2011; Vihma 2014; Walsh 2014; Cohen et al. 2014 and references therein).

The influence of Arctic sea ice on mid-latitude climate has been addressed by evaluating observation data (e.g. Overland et al. 2011; Francis and Vavrus 2012; Jaiser et al. 2012; Screen and Simmonds 2013; Tang et al. 2013) and by performing idealized experiments with atmospheric models (e.g. Deser et al. 2007, 2010; Semmler et al. 2012; Screen et al. 2013; Peings and Magnusdottir 2014).

Previous studies have shown that even though the Arctic sea ice extent declined most strongly in summer the atmospheric response is strongest during winter. From the review paper by Vihma (2014) it becomes clear that most previous studies focused on the lagged impact of summer/ autumn sea ice conditions on the winter large scale circulation while Semmler et al. (2012) and Tang et al. (2013) argue that changes in the meridional winter surface temperature gradient and the associated baroclinicity are important influencing factors. A reduced Arctic summer and autumn sea ice extent favours a higher winter geopotential 
height in the free troposphere over the Arctic and weakened westerly flow over the mid-latitudes (Jaiser et al. 2012). A warmer Arctic winter surface temperature consistent with less sea ice has actually been shown to have a similar effect (Semmler et al. 2012). Regarding the lagged response, Blüthgen et al. (2012) do not see any significant large-scale circulation changes in atmospheric model simulations prescribing the record low Arctic sea ice concentrations from 2007 and associated Arctic and sub-Arctic sea surface temperatures.

While progress in our understanding of Arctic sea ice decline on the middle latitudes has certainly been made, challenges remain. Observational studies, for example, suffer from relatively short time series of reliable satellite measurements of Arctic sea ice extent. Furthermore, it is difficult from observations alone to disentangle cause and effect. In principle, models can and have been used to carry out controlled experiments. However, models are far from perfect in representing atmospheric key features such as Arctic boundary layer inversions and micro-physics. Furthermore, from model studies of the equilibrium response to Arctic sea ice decline it is difficult to understand the physics behind the reponse. This is because in long integrations various processes had time to interact, possibly over vast distances.

In order to get a better understanding of the atmospheric reponse to changes in model formulation or external forcing, Rodwell and Jung (2008) have proposed to study the fast atmospheric response (hours to days) together with the more commonly considered equilibrium response (several months). They argue that by looking at the fast atmospheric adjustment taking different dynamical and physical processes into account, a more thorough understanding of the response can be gained. Here we follow this approach by focussing on the fast atmospheric adjustment to a sudden surface temperature forcing that effectively mimics a decrease in Arctic sea ice thickness.

It should be mentioned that a similar approach has been employed by Deser et al. (2007), they consider the transient seasonal atmospheric adjustment to SST and sea ice extent anomalies restricted to the northern North Atlantic. Diabatic heating of the lower troposphere is found to be associated with surface heat flux anomalies generated by the imposed thermal forcing. This generates a baroclinic response during the first two to three weeks, after which the response becomes mostly equivalent-barotropic. The equilibrium response is reached after 2-2.5 months.

Most previous modelling studies have concentrated on the impact of reduced sea ice extent. However, it is not only the ice extent and concentration but also thickness of Arctic sea ice which has declined substantially over the last few decades (Kwok and Rothrock 2009). Reduced sea ice thickness leads to increased near-surface air temperatures due to an enhanced flux of heat from the relatively warm ocean through the sea ice into the atmosphere. Here we consider the atmospheric response to reduced sea ice thickness all over the Arctic in order to study this effect on the atmospheric circulation. To our knowledge the only previous study focussing on the atmospheric impact of pan-Arctic sea ice thickness reduction is that by Gerdes (2006).

In summary, our study differs from most previous studies in three aspects: Firstly, we investigate the impacts of thinner Arctic sea ice as opposed to reduced Arctic sea ice extent on the Arctic and Northern mid-latitude atmosphere. Secondly, we focus on the fast response to winter as opposed to summer/autumn sea ice conditions excluding any processes acting on longer time scales. Thirdly, instead of studying only the equilibrium response from long climate integrations as has been done in most previous studies, we consider the fast adjustment as well, including the first few time steps of the integration. This enables us to study physical mechanisms of the transient response to a sudden thinning of Arctic sea ice until the equilibrium response is reached. Differences in initial temperature tendencies are split into different physical processes (dynamics, boundary layer turbulence, radiation, large-scale precipitation and convective precipiation) to gain a more thorough physical understanding of the atmospheric response.

The paper is organized as follows: Sect. 2 details the model simulations and Sect. 3 describes results of the main set of 15- and 90-day model ensemble simulations. Discussion and conclusions with regard to previous studies are given in Sect. 4.

\section{Methods}

We used a recent version (cycle 37r3) of the Integrated Forecasting System (IFS), an operational weather forecast model developed at the European Centre for MediumRange Weather Forecasts (ECMWF), for our experiments. The model was run with a time step of $1 \mathrm{~h}$ at a spectral resolution of $\mathrm{T}_{L} 159$, which corresponds to a horizontal resolution of about $125 \mathrm{~km}$, with 91 irregularly spaced vertical levels extending up to $0.01 \mathrm{hPa}$.

15-day forecast experiments were initialized on the 15th of December, 15th of January and 15th of February at 00, 06, 12 and 18 UTC for each of the years from 1979 to 2012, respectively, using ERA-Interim reanalysis data (Dee et al. 2011). This resulted in an ensemble of 408 pairs. Each pair consists of a control (CTL) and a reduced sea ice thickness (RED) simulation. The ensemble mean difference represents the response. In order to provide more detailed insight into the atmospheric response to reduced Arctic sea ice thickness, temperature tendencies due to different physical processes such as vertical diffusion, radiation, 


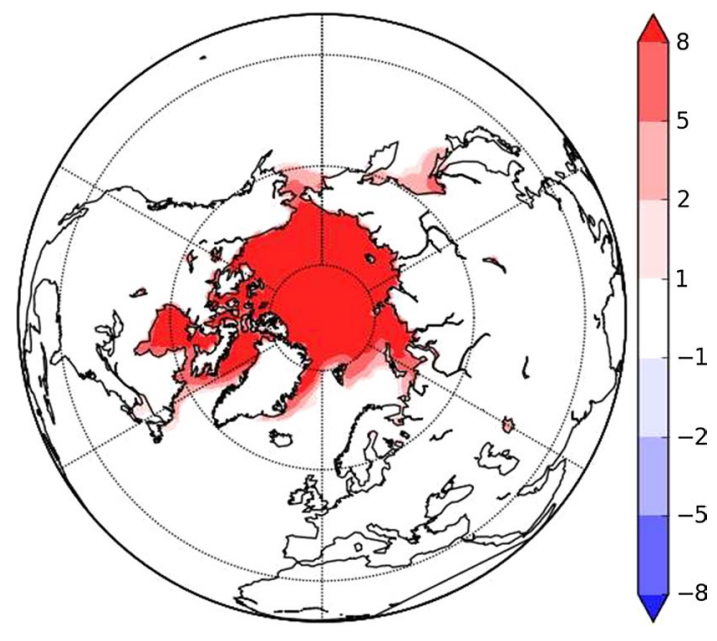

Fig. 1 Mean surface temperature difference $[\mathrm{K}]$ between the ensemble of reduced Arctic sea ice thickness (RED) and control experiments (CTL) $6 \mathrm{~h}$ after initialization

dynamics, convective and large-scale precipitation were postprocessed and archived.

In order to get the quasi-equilibrium response, we initialized additional pairs of 90 day forecast experiments at 00 UTC on the 1st and 15th of November, December and January for each of the years from 1979 to 2012, respectively. This resulted in an ensemble of 204 pairs. Due to data storage limitations temperature tendencies in these long simulations were not archived. For the rest of the paper, results are shown from the 15-day forecast experiments whenever only the first 15 days or a part of them are considered. When 30 days or more are considered, results from the 90-day forecast experiments are shown.

In the CTL simulations we prescribed the sea surface temperature (SST), sea ice surface temperature (SIST) and sea ice concentration from ERA-Interim data. In the RED simulations we increased the SIST by $10 \mathrm{~K}$ throughout the duration of the simulations to reflect a thinning of the sea ice and/or an increased fraction of leads. If the freezing point of sea ice had been exceeded by this SIST increase, SIST has been set to the freezing point of sea water. The prescribed mean surface temperature forcing, i.e. the ensemble difference RED minus CTL simulations, is shown in Fig. 1.

For typical Arctic sea ice thicknesses of 1-2 $\mathrm{m}$ this surface temperature difference resembles a reduction of Arctic sea ice thickness by about 60-70\% if leaving out any other influencing factors. This can be derived from the equilibrium response study by Semmler et al. (2012) when considering their winter average Arctic SIST and their residual surface energy budget from the reference and reduced sea ice simulations. Comparable, if not larger sea ice thickness reductions are simulated in coupled model experiments for the end of this century compared to the end of last century with a medium to high radiative forcing (e.g. Deser et al. 2010).

Another possible way how a $10 \mathrm{~K}$ SIST increase could be realized is through larger leads or a combination of thinner sea ice and larger leads. According to Lüpkes et al. (2008), for sea ice concentrations of more than $90 \%$ commonly observed in winter in the Arctic, small increases in the fraction of leads can lead to substantial temperature increases. Furthermore, a $10 \mathrm{~K}$ near-surface Arctic Ocean winter temperature increase is typically simulated for the end of this century in state-of-the art coupled climate model simulations from the Coupled Model Intercomparison Project 5 (CMIP5) with a medium to high radiative forcing (e.g. Koenigk et al. 2013; Vavrus et al. 2012).

Notice that our study and the one by Semmler et al. (2012) are unique in the sense that SIST is specified; in other studies the SIST freely develops through the surface energy balance.

\section{Results}

To start with, Fig. 2 shows the development of zonally averaged mean temperature anomalies over a 15 day period after the sudden thinning of the Arctic sea ice along with climatological mean values from the CTL simulation. A temperature response of moderate amplitude $(2-2.5 \mathrm{~K})$ spreads throughout the Arctic boundary layer within the first day of the experiment. After that the near surface response further increases until saturation is reached 5-10 days into the integration at a value of about $6 \mathrm{~K}$. The temperature response slowly "propagates" into the free troposphere over the Arctic with values reaching $0.5-2 \mathrm{~K}$ after 10 days or so. The temperature response is such as to decrease the meridional temperature gradient north of about $60^{\circ} \mathrm{N}$. Notice, that this is way north of the main baroclinic zones in the storm formation regions of the North Atlantic and North Pacific suggesting that the fast atmospheric response to Arctic sea ice thinning has a minor influence on the midlatitude storm tracks. Given the strong height dependence of the temperature response, our results also highlight the importance of properly representing Arctic boundary layer processes in models.

Figure 3 shows how temperature tendencies of different atmospheric processes in the boundary layer respond to reduced sea ice thickness during the first 5 days of the simulation. The tendencies for days 6-10 and 11-15 are not shown since they are very similar to the ones for days $1-5$. The first thing to notice is that vertical diffusion (i.e. boundary layer turbulence) and radiation are the major contributors to the boundary layer warming during the first $6 \mathrm{~h}$ of the simulation (Fig. 3, left column). This can be 
Fig. 2 Mean vertical temperature profiles for CTL (black contour lines), interval $\left.4\left[{ }^{\circ} \mathrm{C}\right]\right)$ and differences (colour shading, $[\mathrm{K}]$ ) between RED and CTL averaged over a the first $6 \mathrm{~h}, \mathbf{b}$ the first day, $\mathbf{c}$ over the first 2 days, $\mathbf{d}$ days $1-5$, e days 6-10 and $\mathbf{f}$ days 11-15. Differences are only shown when they are significant at the $95 \%$ level according to a Wilcoxon test
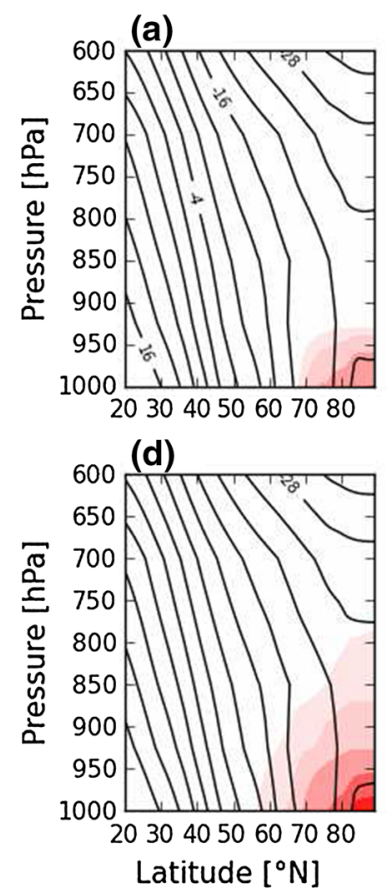
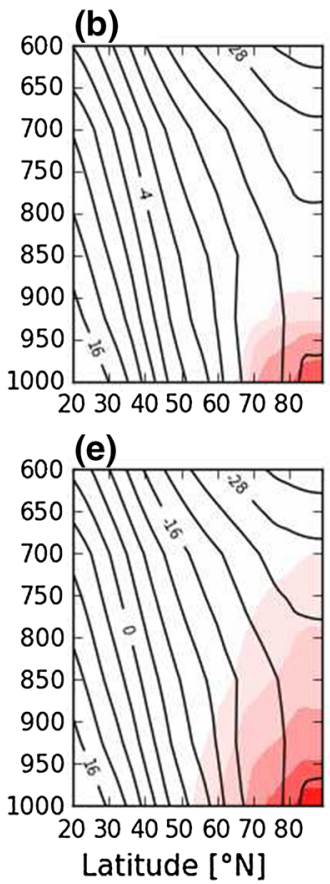

(c)

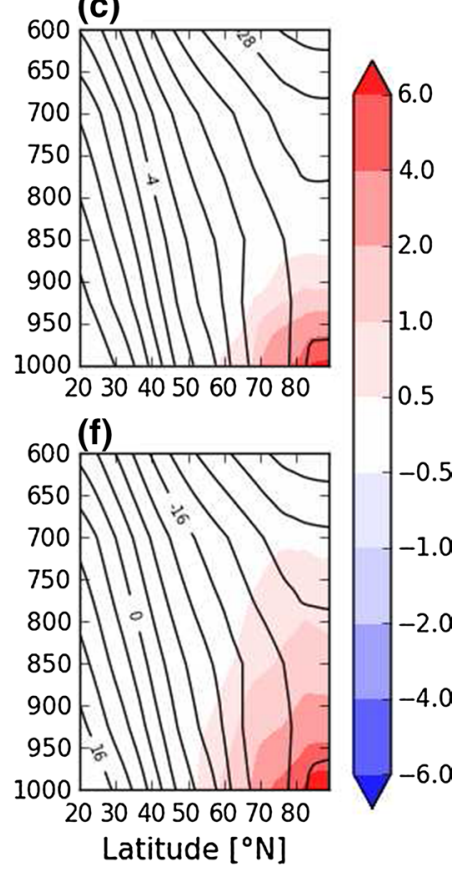

explained by the warmer surface which leads to anomalous upward turbulent heat flux and longwave radiation; $6 \mathrm{~h}$ into the integration, the dynamics, convection and large-scale precipitation all play a negligible role.

A different picture emerges beyond $6 \mathrm{~h}$. Most notably, 1-5 days into the integration the dynamics (i.e. temperature advection) is starting to play an increasingly important role in balancing the anomalous temperature tendencies due to vertical diffusion. Both equatorward and poleward temperature advection in the boundary layer around the ice edge becomes weaker (negative and positive, respectively) due to the reduced temperature contrast between sea ice and open water (Fig. 3h-i). The associated reduction in turbulent sensible heat flux out of the ocean is consistent with a reduced heating due to vertical diffusion (Fig. 3c). The reduction of the radiative heating response further into the integration results from the fact that the increasingly warmer air starts to emit more longwave radiation which counteracts the anomalous radiative heating from below.

Throughout the first 5 days anomalous temperature tendencies due to convection and large-scale precipitation are relatively small and associated with the ice edge zone where the meridional temperature gradient is changed. Generally, thinner Arctic sea ice is accompanied by more convective heating and less large-scale precipitation. This is especially true north of the ice edge which is plausible due to the reduced stability. Above the boundary layer qualitatively similar anomalous tendencies are simulated but they are one order of magnitude smaller than in the boundary layer (not shown). It makes sense that changes are largely restricted to the boundary layer because of the strong vertical stability in winter.

Changes in the near-surface and mid-troposphere largescale circulation are shown in Figs. 4 and 5, respectively. Increased SIST causes a negative mean sea level pressure anomaly along with a positive anomaly in the $500 \mathrm{hPa}$ geopotential height over the Arctic sea ice area. While the negative mean sea level pressure anomaly develops very quickly and starts to diminish after 15 days over the Eastern Arctic, the positive $500 \mathrm{hPa}$ geopotential height anomaly slowly builds up over the course of the 90 days. The initial baroclinic response is consistent with what would be expected from an anomalous near-surface heating.

Interestingly, already within the first 5 days a positive equivalent-barotropic atmospheric response develops in some areas including north-western Siberia, northern Europe, the north-eastern North Atlantic, and the northeastern North Pacific. However, the barotropic response is still relatively small albeit significant at the $95 \%$ level. While over the north-eastern North Pacific, northern Europe, and the north-eastern North Atlantic the barotropic response becomes insignificant and even changes sign over the north-eastern North Pacific, the positive anticyclonic barotropic response over north-western Siberia remains robust over the entire 90 days.

Next we turn our attention to the impacts of a sudden sea ice thinning on synoptic activity. Here, the approach of Jung (2005) is used, which is based on taking the standard deviation of 1-day changes of $500 \mathrm{hPa}$ geopotential height. The results for CTL (Fig. 6a) compare well with corresponding results for ERA-40 reanalysis data (Jung 2005, 
Fig. 3 Differences in mean temperature tendencies $[\mathrm{K} /$ day] between RED and CTL for different atmospheric processes vertically averaged from the surface to $925 \mathrm{hPa}$. Results are shown for a-c vertical diffusion and orographic gravity waves, d-f radiation, $\mathbf{g - i}$ dynamics, $\mathbf{j}-\mathbf{l}$ convective precipitation, $\mathbf{m}-\mathbf{o}$ large-scale precipitation and different forecast lead times: (left column) first $6 \mathrm{~h}$, (middle column) first day, and (right column) days 1-5. For details see text (a)

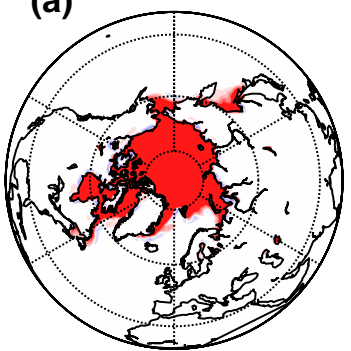

(d)

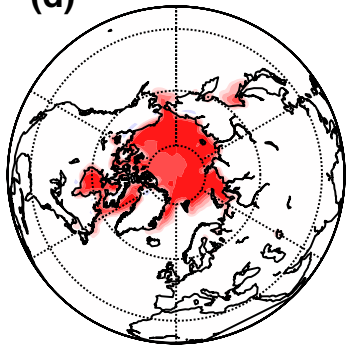

(g)

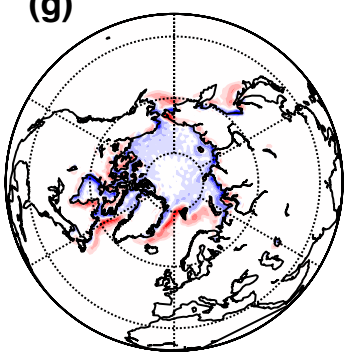

(j)

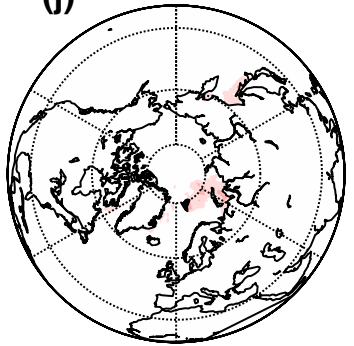

(m)

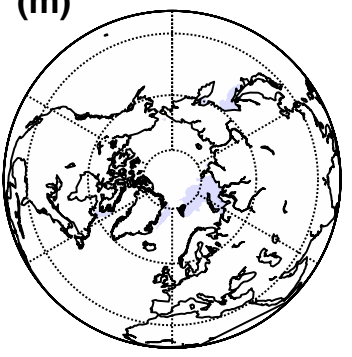

(b)

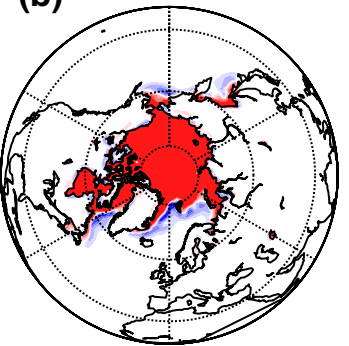

(e)

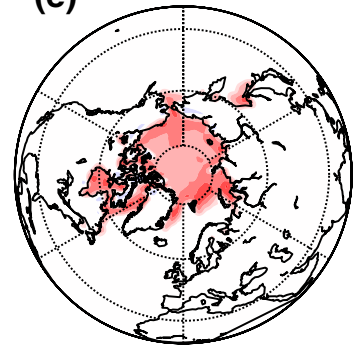

(h)

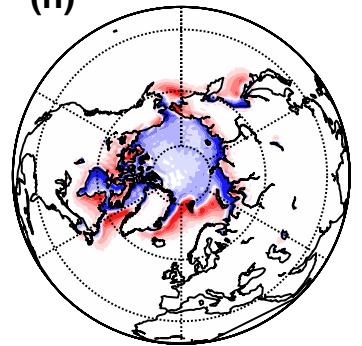

(k)

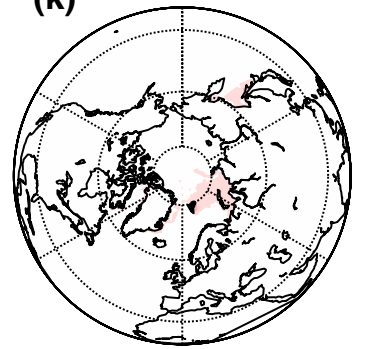

(n)

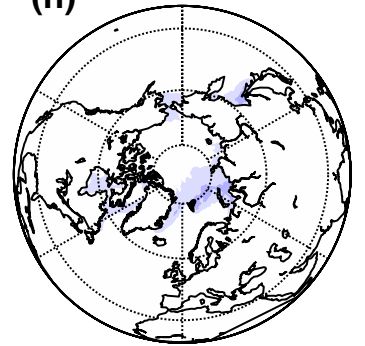

(c)

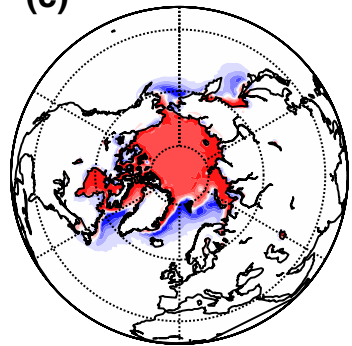

(f)

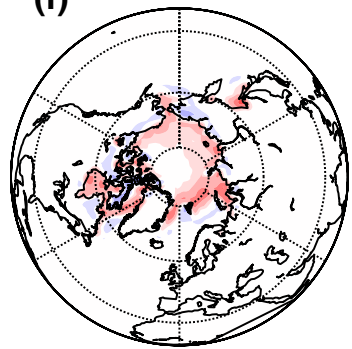

(i)

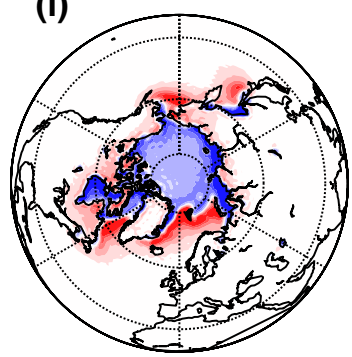

(I)

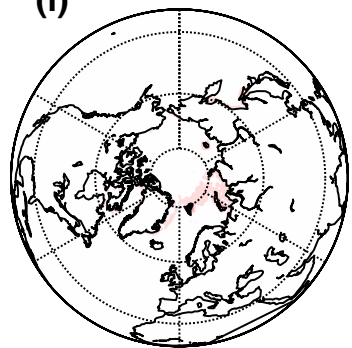

(o)

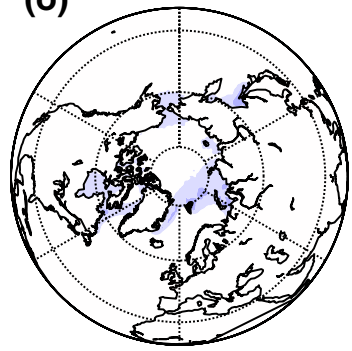

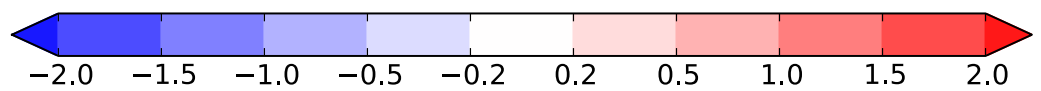

their Fig. 12). The response of synoptic activity in RED to a sudden thinning of the Arctic sea ice can be inferred from Fig. 7. Generally, a decrease in synoptic activity can be found which is strongest over the central Arctic and extends into sub-Arctic areas. This decrease intensifies over time.
Two competing effects could influence the change in synoptic activity. Firstly, according to the Eady index (Eady 1949), reduced static stability potentially leads to increased synoptic activity. Secondly, the reduced meridional temperature gradient between $60-80^{\circ} \mathrm{N}$ should lead to 
Fig. 4 Mean sea level pressure response $(\mathrm{hPa})$ for $\mathrm{RED}$ compared to CTL averaged over forecast days a 1-5, b 1-15, c 1-30, and d 31-90. Stippled areas are significant at the $95 \%$ level according to a Wilcoxon test. Values are only shown for grid points where the orography is below $1000 \mathrm{~m}$ above sea level to exclude unrealistic values due to extrapolation
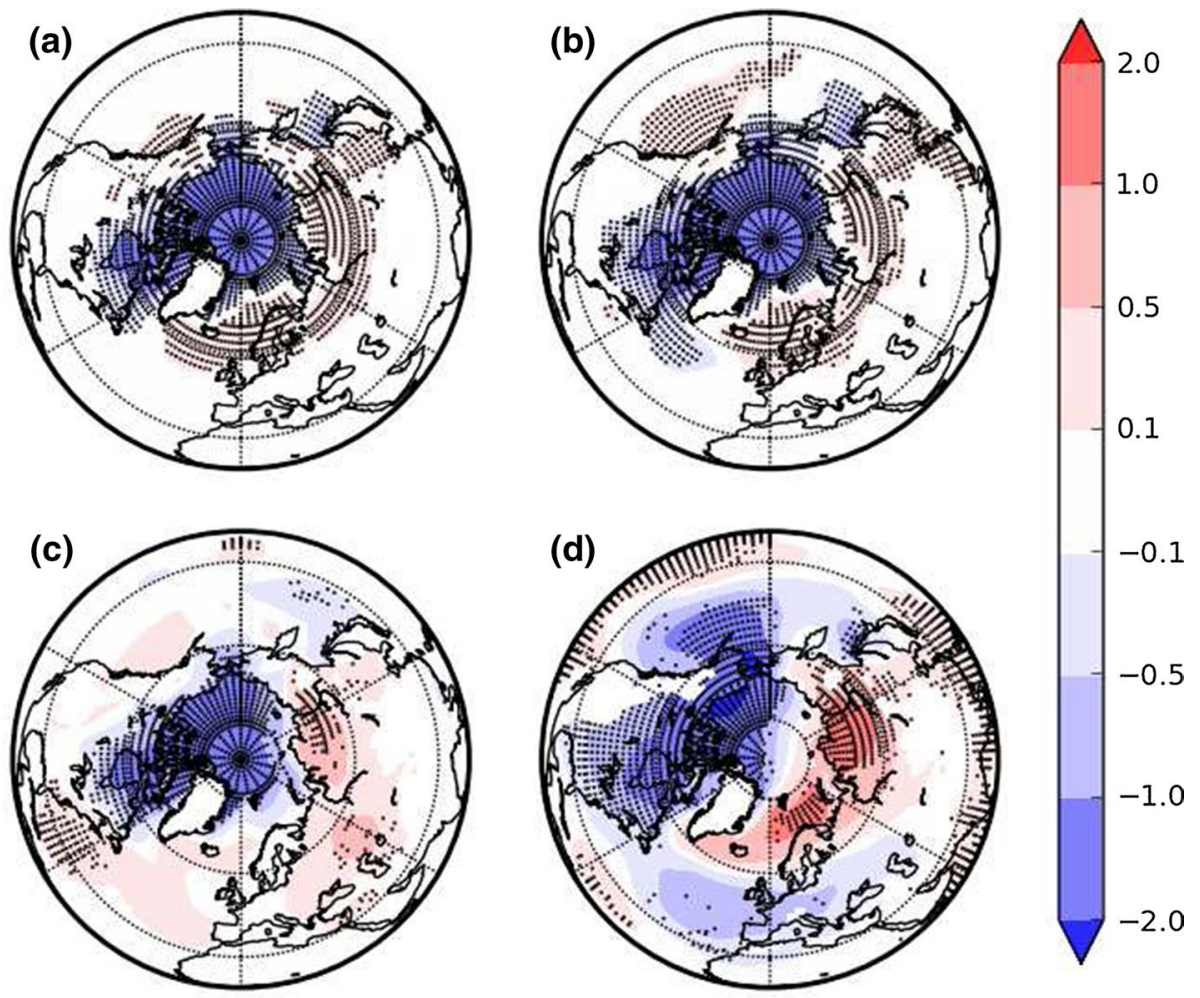
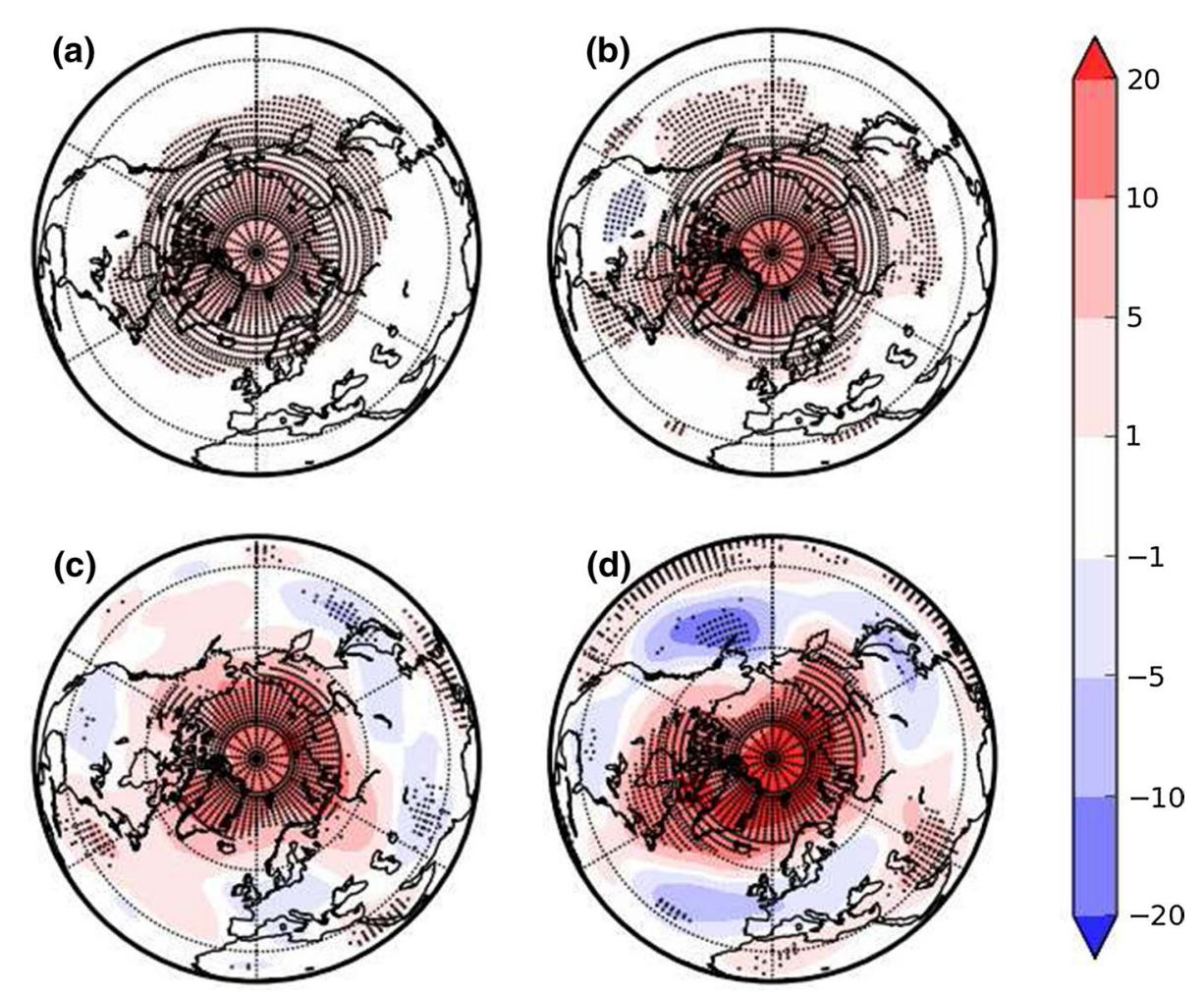

Fig. 5 Same as in Fig. 4, except for $500 \mathrm{hPa}$ geopotential height fields $(\mathrm{m})$. Values are only shown for grid points where the orography is below $5000 \mathrm{~m}$ above sea level to exclude unrealistic values due to extrapolation reduced synoptic activity-even though the largest temperature changes occur in the boundary layer. The maximum Eady growth rate from the ensemble of CTL simulations is shown in Fig. 6b, the response of the maximum Eady growth rate to the thin Arctic sea ice in Fig. 8. In the first 5 days there is a significant positive response in the 
Fig. 6 a Standard deviation of highpass-filtered daily 500 $\mathrm{hPa}$ geopotential height fields ('synoptic activity' according to Jung 2005 in $\mathrm{m}$ ), b maximum Eady growth rate between 500 and $850 \mathrm{hPa}$ based on 6-hourly data $\left(\right.$ day $\left.^{-1}\right)$ according to Eady (1949), and $\mathbf{c} \mathrm{E}$ vector $\left(\mathrm{m}^{2} / \mathrm{s}^{2}\right.$, vector arrows) and its divergence $\left(\mathrm{m} / \mathrm{s}^{2}\right.$, colour shading) for highpass transients at 250 $\mathrm{hPa}$ according to Hoskins et al. (1983) in the ensemble of CTL simulations for forecast days $1-15$. For details see text
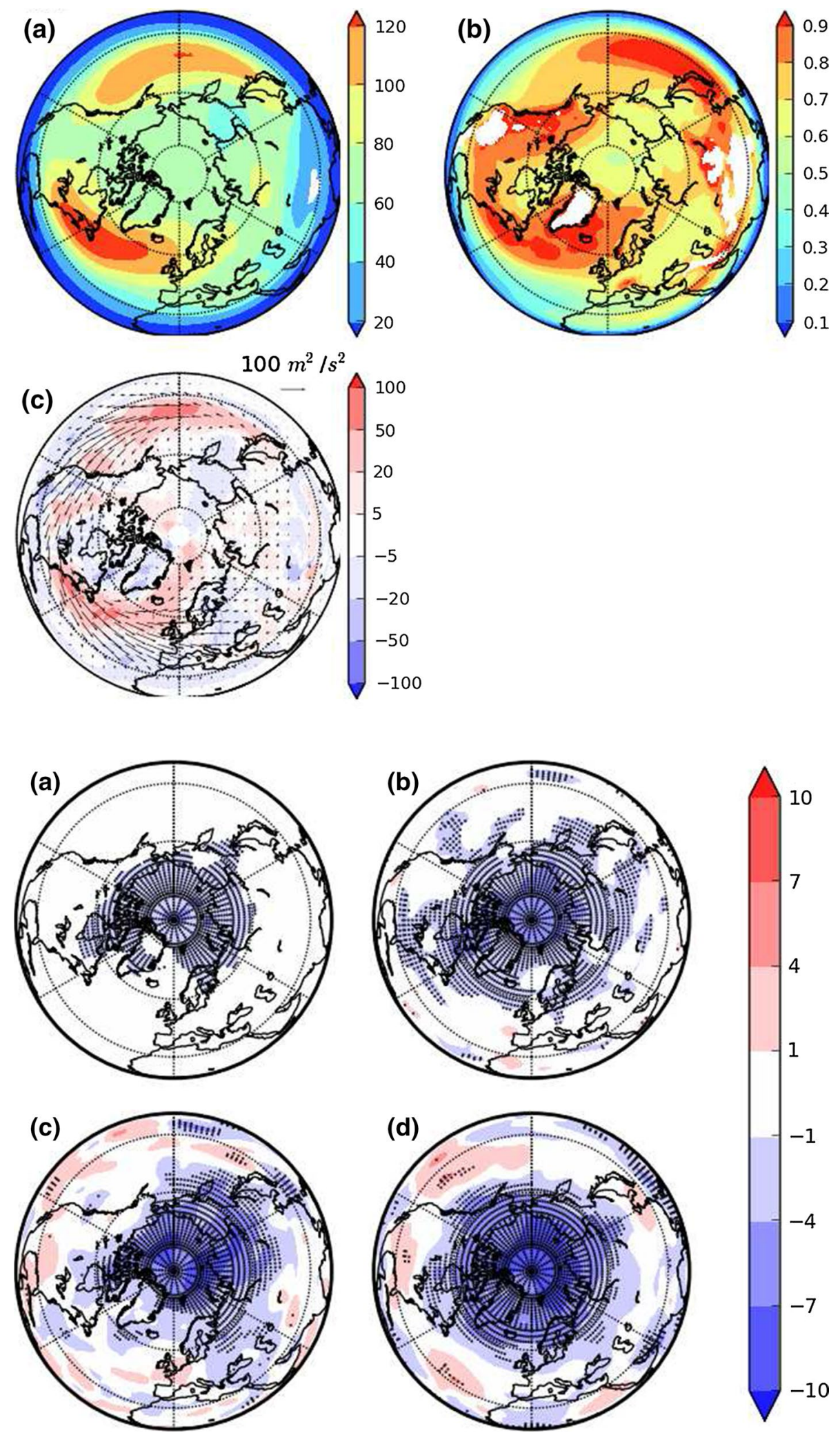

Fig. 7 Relative change in the standard deviation of highpassfiltered daily $500 \mathrm{hPa}$ geopotential height fields (\%) for RED compared to CTL, forecast days a $1-5$, b $1-15, \mathbf{c} 1-30$ and d 31-90. Stippled areas are significant at the $95 \%$ level according to a Wilcoxon test. Values are only shown for grid points where the Earth's surface is below $5000 \mathrm{~m}$ above sea level to exclude unrealistic values due to extrapolation

the negative synoptic activity response shows that the Eady growth index is not a good measure to describe synoptic activity for the sea ice-covered Arctic. In the areas adjacent maximum Eady growth rate over the Arctic sea ice which becomes insignificant with increasing integration time. The positive maximum Eady growth rate response along with 
Fig. 8 Change in the maximum eady growth rate between 500 and $850 \mathrm{hPa}$ based on 6-hourly data $\left(\right.$ day $\left.^{-1}\right)$ for RED compared to CTL, forecast days a $1-5$, b $1-15$, c $1-30$ and d 31-90. Stippled areas are significant at the $95 \%$ level according to a Wilcoxon test. Values are only shown for grid points where the Earth's surface is below $1500 \mathrm{~m}$ above sea level to exclude unrealistic values due to extrapolation
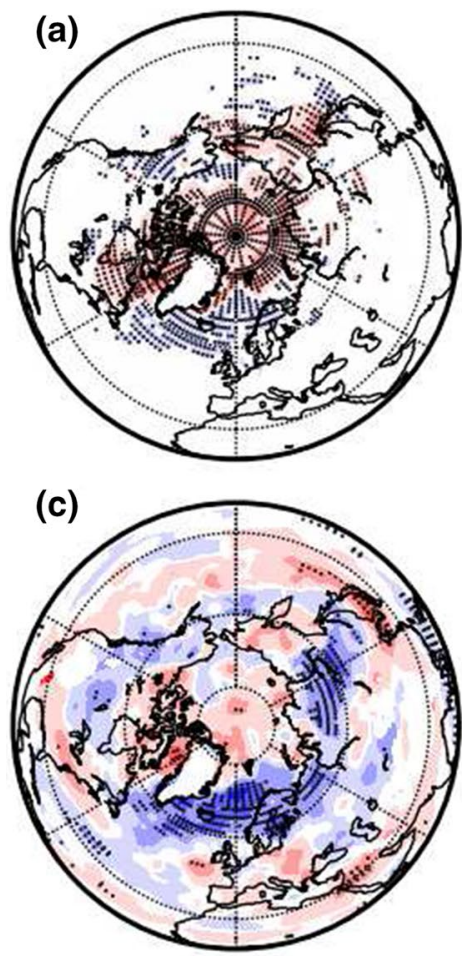
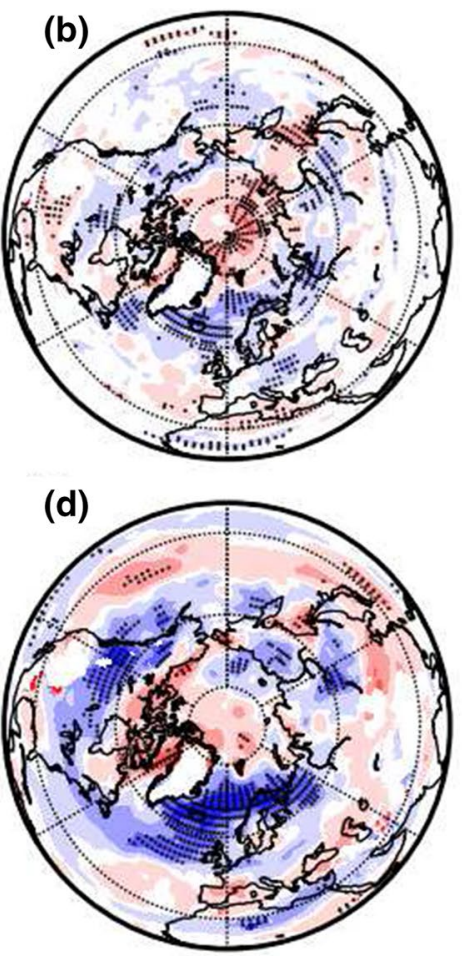

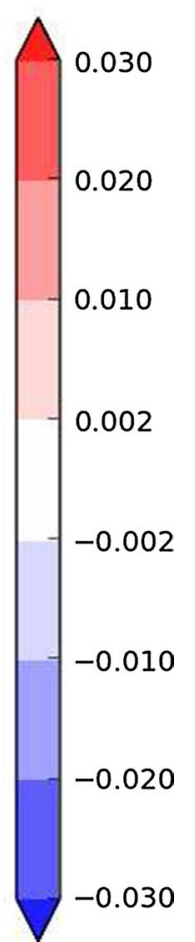

to the Arctic sea ice a negative response in maximum Eady growth rate develops. It becomes significant after about 15 days especially over north-western Siberia, northern Europe, the north-eastern North Atlantic, and north-western North America. The first three areas are the areas where an anticyclonic barotropic atmospheric circulation response is found.

There is another, perhaps less obvious explanation for the change in synoptic activity, which has to do with the fact that high-latitude synoptic activity in the IFS is sensitive to the cloud microphysics used (Jung et al. 2010). Due to the strong changes in the Arctic boundary layer in RED compared to CTL the relative importance of the cloud microphysical scheme might change which in turn might alter Arctic cyclone activity.

An important question is whether anomalous eddy mean flow interaction could cause some of the simulated largescale circulation responses. Figure $6 \mathrm{c}$ shows the E vector for highpass transients according to Hoskins et al. (1983) along with its divergence in the CTL simulations and Fig. 9 the response in the thin Arctic sea ice experiments. In the CTL simulations the North Pacific and North Atlantic storm tracks in which wave energy propagates from the west to the east into the continental areas (E vectors) and intensifies the jet stream due to the eddy mean flow interaction (E-vector divergence) can clearly be identified. Regarding the response to the thinner Arctic sea ice, the changes in divergence are very noisy and hence it is not possible to identify a substantial pattern change in the eddy mean flow interaction. The wave energy propagation seems to be reduced over the north-eastern North Pacific in the first 15 days and accelerated afterwards. Note that also the barotropic circulation response changes sign from anticyclonic to cyclonic after the first 15 days. Over the north-eastern North Atlantic the wave propagation is reduced during the entire 90 days which may explain the anticyclonic barotropic response in this area and downstream of it.

\section{Discussion and conclusions}

There have been numerous recent studies on the influence of Arctic sea ice decline on the atmospheric circulation. Our study differs from most previous ones in three respects. Firstly, the atmospheric response to reduced sea ice thickness rather than area is studied-meaning that a forcing is applied all over the Arctic as opposed to only in the vicinity of the sea ice edge. Secondly, the emphasis is on the fast atmospheric response within the winter season leaving out processes operating on longer time scale. We argue that relatively fast atmospheric processes are capable of invoking a large-scale response. Thirdly, we include a diagnosis of the model's physical processes during the transient adjustment. We argue that this setup helps to unravel the dynamical and physical mechanisms underlying the atmospheric response.

The fast response to reduced Arctic sea ice thickness is largest in the Arctic boundary layer; in the free atmosphere and in the middle latitudes the response is relatively weak 
Fig. 9 Change in the E vector $\left(\mathrm{m}^{2} / \mathrm{s}^{2}\right.$, vector arrows $)$ and its divergence $\left(\mathrm{m} / \mathrm{s}^{2}\right.$, colour shading) for highpass transients at $250 \mathrm{hPa}$ according to Hoskins et al. (1983) for RED compared to CTL, forecast days a $1-5, \mathbf{b}$ $1-15$, c $1-30$ and d $31-90$
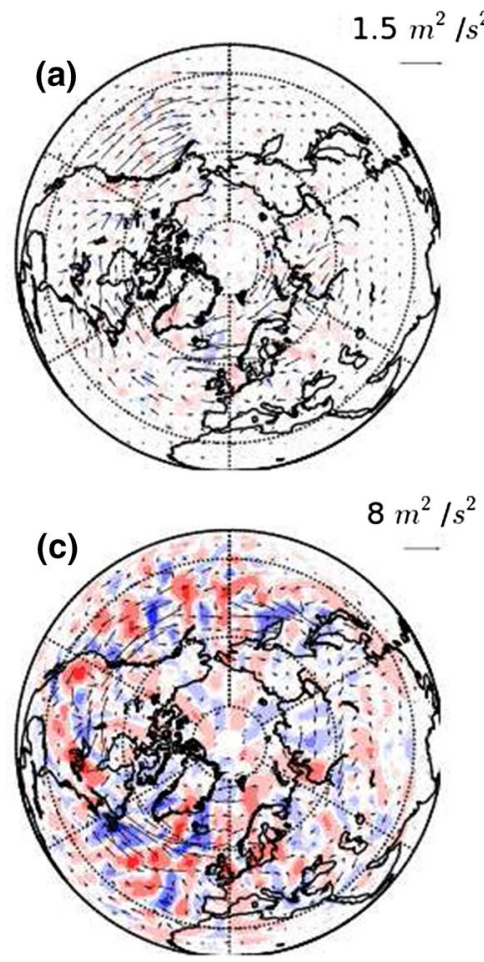

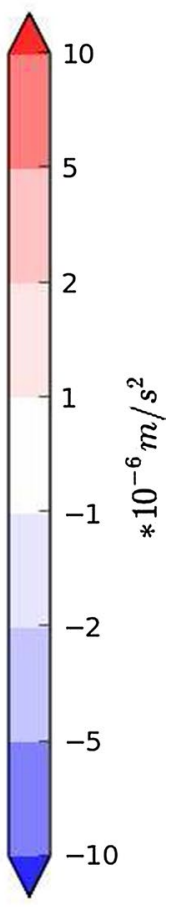

compared to the background level of variability. The relatively weak temperature response in the free atmosphere is presumably a result of strong decoupling due to the presence of a stable boundary layer. The temperature in the Arctic boundary layer responds very quickly to a sudden decline in Arctic sea ice thickness. In this uncoupled setup, saturation of the temperature response has been approximately reached as early as a few days into the integration.

Our results highlight the importance of Arctic boundary layer processes in keeping the response of the large-scale atmospheric circulation to a very strong surface forcing rather limited. In this respect it is a matter of concern that atmospheric circulation models still show substantial shortcomings in simulating stable boundary layers (Holtslag et al. 2013); this is also true for high-resolution weather prediction models which are known to possess too diffusive boundary layers (Sandu et al. 2013).

Our results agree with those by Deser et al. (2010) in the sense that in a quasi-equilibrium state boundary layer turbulence is the most important process in terms of transferring the anomalous surface heat upward and that this effect is balanced by anomalous temperature advection. The fast atmospheric response after 1 day to a sudden thinning of the Arctic sea ice, however, is quite different showing strong anomalous positive temperature tendencies in the Arctic boundary layer due to enhanced longwave radiation from the surface; further into the integration the anomalous temperature tendency due to radiation starts to reduce which is in agreement with results from the equilibrium simulation of Deser et al. (2010) who find a comparably small longwave radiation contribution to the total anomalous temperature tendency.

The main difference between this study and the one by Deser et al. (2010) lies in the fact that in our experiments convective and large scale precipitation plays a minor role, whereas the condensational temperature tendency response is sizable in the latter study. Whether this discrepancy results from the fact that different models are used and/or whether the different experimental setup (reponse to only sea ice thickness reduction versus combined sea ice extent and sea ice thickness reduction) contributes remains to be shown. To resolve this issue conclusively a more coordinated modelling approach would be needed.

In line with the study by Deser et al. (2007), which considers the initial response to positive SST and negative sea ice extent anomalies in the North Atlantic sector, the imposed thermal surface forcing leads to a diabatic heating of the lower troposphere. Likewise, a baroclinic response with negative mean sea level pressure anomalies and positive $500 \mathrm{hPa}$ geopotential height anomalies can be seen in the first few days when the temperature response is still developing. However, due to the thinning of the sea ice over the entire Arctic our initial baroclinic response is not restricted to the sea ice edge but occurs over the entire Arctic-with an emphasis on the western Arctic presumably due to the asymmetry in the land-sea distribution. After a few days, a positive equivalent-barotropic response starts to develop in regions where there is no thermal surface 
forcing, in our case over north-western Siberia, north-eastern Europe and the north-eastern North Atlantic.

Our response is a result of a reduction of the meridional temperature gradient only north of about $60^{\circ} \mathrm{N}$ rather than also in the Northern mid-latitudes as implied by Francis and Vavrus (2012), Jaiser et al. (2012) and other studies based on reanalysis data. We find that reduced winter sea ice thickness goes along with a decrease in the meridional temperature gradient at high latitudes and therefore reduced synoptic activity; the maximum Eady growth rate between 500 and $850 \mathrm{hPa}$ slightly increases due to slightly reduced static stability but this increase is not reflected in the level of synoptic activity. Reduced synoptic activity is consistent with a decreased storminess reported by Seierstad and Bader (2009). The changes already arise after a few days into the integration and are largely restricted to the Arctic itself. The positions of the major storm tracks over the North Atlantic and North Pacific remain unaffected, at least up to about 15 days into the integration. Over continental areas such as north-western Siberia and north-eastern Europe the majority of cyclones is quasi-barotropic. Reduced wave energy propagation associated with synoptic eddies from the northeastern North Atlantic into Northern Europe and northwestern Siberia could lead to fewer cyclones and therefore the large-scale anticyclonic equivalent-barotropic response. These results are consistent with Tang et al. (2013) who studied the impact of decreased winter sea ice from reanalysis data and showed that fast purely tropospheric responses such as fewer cyclones within the winter season can cause the described large-scale circulation changes.

A positive equivalent-barotropic response over northwestern Siberia and north-eastern Europe has also been found in idealized equilibrium experiments by Semmler et al. (2012), Deser et al. (2010) and Gerdes (2006) and seems to be a robust feature during the whole winter. A relatively weak and inconsistent response over the North Atlantic and North Pacific along with a larger response over the Northern Hemisphere continents is consistent with the results of Jung et al. (2014) from sub-seasonal forecast experiments with and without relaxation of the Arctic atmosphere towards reanalysis data.

Various other previous studies such as Petoukhov and Semenov (2010), Liu et al. (2012), Orsolini et al. (2012), Jaiser et al. (2012) and Jaiser et al. (2013) link changes in winter circulation with low August/September sea ice concentration. They argue that the winter circulation is affected by modified planetary wave activity caused by the low August/ September sea ice concentration which may impact stratosphere circulation that may in turn propagate down to the troposphere. Downward propagation of stratospheric anomalies happens on a time scale of weeks (Baldwin and Dunkerton 2001) and two-way stratosphere-troposphere interactions even on a time scale of weeks to months (e.g. Cohen et al.
2009; Petoukhov and Semenov 2010; Orsolini et al. 2012). Walsh (2014) note that the Arctic-midlatitude linkage through the stratosphere is still not firmly established. Furthermore, Blüthgen et al. (2012) do not note any significant large-scale circulation changes in atmospheric model simulations with 2007 Arctic sea ice and sea surface temperature conditions.

Our results suggest that it is not necessary to invoke large-scale processes such as stratosphere-troposphere interaction to cause large-scale winter circulation changes but that relatively fast tropospheric processes with a time scale of only a few days are sufficient. One could think that in such a case it is not possible to benefit from predictability of Northern mid-latitude large scale winter circulation due to late summer/autumn sea ice conditions. A link might still be present, however, because the winter sea ice itself may show some predictability based on the late summer/autumn sea ice. Furthermore, the results show that the response amplifies over the course of the 90 days. This leaves some room for relatively slow atmospheric processes such as troposphere-stratosphere interactions to play a role in intensifying the response-our results imply, however, that fast atmospheric processes are key in initiating the response.

Acknowledgments The authors acknowledge ECMWF for providing the supercomputing resources under the ECMWF special project SPDEJUNG2. S.S. benefited from funding through the Helmholtz Climate Initiative REKLIM. The authors appreciate helpful and valuable comments from two anonymous reviewers which helped to improve the manuscript.

Open Access This article is distributed under the terms of the Creative Commons Attribution 4.0 International License (http://creativecommons.org/licenses/by/4.0/), which permits unrestricted use, distribution, and reproduction in any medium, provided you give appropriate credit to the original author(s) and the source, provide a link to the Creative Commons license, and indicate if changes were made.

\section{References}

Bader J, Mesquita MD, Hodges KI, Keenlyside N, Østerhus S, Miles M (2011) A review on Northern Hemisphere sea-ice, storminess and the North Atlantic oscillation: observations and projected changes. Atmos Res 101(4):809-834

Baldwin MP, Dunkerton TJ (2001) Stratospheric harbingers of anomalous weather regimes. Science 294(5542):581-584

Blüthgen J, Gerdes R, Werner M (2012) Atmospheric response to the extreme Arctic sea ice conditions in 2007. Geophys Res Lett 39(L02):707

Budikova D (2009) Role of Arctic sea ice in global atmospheric circulation: a review. Glob Planet Change 68(3):149-163

Cohen J, Barlow M, Saito K (2009) Decadal fluctuations in planetary wave forcing modulate global warming in late boreal winter. J Clim 22(16):4418-4426

Cohen J, Screen JA, Furtado JC, Barlow M, Whittleston D, Coumou D, Francis J, Dethloff K, Entekhabi D, Overland J, Jones 
J (2014) Recent Arctic amplification and extreme mid-latitude weather. Nat Geosci 7(9):627-637

Deser C, Robert Tomas A, Peng S (2007) The transient atmospheric circulation response to North Atlantic SST and sea ice anomalies. J Clim 20:4751-4767

Deser C, Tomas R, Alexander M, Lawrence D (2010) The seasonal atmospheric response to projected Arctic sea ice loss in the late twenty-first century. J Clim 23(2):333-351

Eady ET (1949) Long waves and cyclone waves. Tellus 1:33-52

Francis JA, Vavrus SJ (2012) Evidence linking Arctic amplification to extreme weather in mid-latitudes. Geophys Res Lett 39(6):L06,801

Gerdes R (2006) Atmospheric response to changes in Arctic sea ice thickness. Geophys Res Lett 33(L18):709

Holtslag A, Svensson G, Baas P, Basu S, Beare B, Beljaars A, Bosveld F, Cuxart J, Lindvall J, Steeneveld G et al (2013) Stable atmospheric boundary layers and diurnal cycles: challenges for weather and climate models. Bull Am Meteorol Soc 94(11):1691-1706

Hoskins BJ, James IN, White GH (1983) The shape, propagation and mean-flow interaction of large-scale weather systems. J Atmos Sci 40(7):1595-1612

Jaiser R, Dethloff K, Handorf D, Rinke A, Cohen J (2012) Impact of sea ice cover changes on the Northern Hemisphere atmospheric winter circulation. Tellus A 64(11):595. doi:10.3402/tellusa. v64i0.11595

Jaiser R, Dethloff K, Handorf D (2013) Stratospheric response to Arctic sea ice retreat and associated planetary wave propagation changes. Tellus A 65(19):375

Jung $T$ (2005) Systematic errors of the atmospheric circulation in the ECMWF forecasting system. Q J R Meteorol Soc 131:1045-1073

Jung T, Balsamo G, Bechtold P, Beljaars A, Köhler M, Miller M, Morcrette JJ, Orr A, Rodwell M, Tompkins A (2010) The ECMWF model climate: recent progress through improved physical parametrizations. Q J R Meteorol Soc 136(650):1145-1160

Jung T, Kasper MA, Semmler T, Serrar S (2014) Arctic influence on medium-range and extended-range prediction in mid-latitudes. Geophysical Research Letters 41. doi:10.1002/2014GL059961

Koenigk T, Brodeau L, Graversen RG, Karlsson J, Svensson G, Tjernström M, Willén U, Wyser K (2013) Arctic climate change in 21st century CMIP5 simulations with EC-Earth. Clim Dyn 40(11-12):2719-2743

Kwok R, Rothrock D (2009) Decline in Arctic sea ice thickness from submarine and ICESat records: 1958-2008. Geophys Res Lett 36(15):L15,501

Liu J, Curry JA, Wang H, Song M, Horton RM (2012) Impact of declining Arctic sea ice on winter snowfall. Proc Nat Acad Sci 109(11):4074-4079
Lüpkes C, Vihma T, Birnbaum G, Wacker U (2008) Influence of leads in sea ice on the temperature of the atmospheric boundary layer during polar night. Geophys Res Lett 35(3):L03,805

Orsolini YJ, Senan R, Benestad RE, Melsom A (2012) Autumn atmospheric response to the 2007 low Arctic sea ice extent in coupled ocean-atmosphere hindcasts. Clim dyn 38(11-12):2437-2448

Overland JE, Wood KR, Wang M (2011) Warm Arctic-cold continents: climate impacts of the newly open Arctic sea. Polar Res 30(1):157-187. doi:10.3402/polar.v30i0.15787

Parkinson CL, Comiso JC (2013) On the 2012 record low Arctic sea ice cover: combined impact of preconditioning and an August storm. Geophys Res Lett 40(7):1356-1361

Peings Y, Magnusdottir G (2014) Response of the wintertime Northern Hemisphere atmospheric circulation to current and projected Arctic Sea Ice Decline: a numerical study with CAM5. J Clim 27(1):244-264

Petoukhov V, Semenov VA (2010) A link between reduced BarentsKara sea ice and cold winter extremes over northern continents. J Geophys Res Atmos (1984-2012) 115:D21-111

Rodwell MJ, Jung T (2008) Understanding the local and global impacts of model physics changes: an aerosol example. Q J R Meteorol Soc 134:1479-1497

Sandu I, Beljaars A, Bechtold P, Mauritsen T, Balsamo G (2013) Why is it so difficult to represent stably stratified conditions in numerical weather prediction (NWP) models? J Adv Model Earth Syst 5(2):117-133

Screen JA, Simmonds I (2013) Exploring links between Arctic amplification and mid-latitude weather. Geophys Res Lett 40(5):959-964

Screen JA, Simmonds I, Deser C, Tomas R (2013) The atmospheric response to three decades of observed Arctic sea ice loss. J Clim 26(4):1230-1248

Seierstad IA, Bader J (2009) Impact of a projected future Arctic sea ice reduction on extratropical storminess and the NAO. Clim dyn 33(7-8):937-943

Semmler T, McGrath R, Wang S (2012) The impact of Arctic sea ice on the Arctic energy budget and on the climate of the Northern mid-latitudes. Clim Dyn 39(11):2675-2694

Tang Q, Zhang X, Yang X, Francis JA (2013) Cold winter extremes in northern continents linked to Arctic sea ice loss. Environ Res Lett 8(1):0140,36

Vavrus SJ, Holland MM, Jahn A, Bailey DA, Blazey BA (2012) Twenty-first-century Arctic climate change in CCSM4. J Clim 25:2696-2710

Vihma T (2014) Effects of Arctic sea ice decline on weather and climate: a review. Surveys in Geophysics, pp 1-40

Walsh JE (2014) Intensified warming of the Arctic: causes and impacts on middle latitudes. Glob Planet Change 117:52-63 